\title{
DELIBERATIONS ON LEAF VENATION AS SUPPORTED BY THE ANNONACEAE*
}

\author{
Edward P. Klucking** \\ INTRODUCTION
}

Much of our knowledge of the past record of angiosperms depends upon the identification and description of fossil leaf remains. Efforts to identify and describe these fossil leaves are greatly limited by insufficient familiarity with the nature, diversitv, and distribution of leaf venation patterns among extant angiosperm families. They are also hampered by the lack of a suitable terminology for describing venational features. Because of these shortcomings, many Tertiary paleobotanists have begun extensive studies of the leaves of extant angiosperm plants. Such a study of the Annonacear has suggested some interesting aspects of leaf venation.

The annonaceae is a relatively large family consisting of 130 genera and 2300 species (Walker. 1971). It is the largest family of the Magnoliales, an order including 19 families, 242 genera, and 5477 species of the most primitive flowering plants (Cronquist, 1968). All of the members of the Annonaceac are woody in habit and nearly alwave oceur at low elevations in the tropical areas of Southeast Asia, Central and South America, and Africa. The leaves of these plants are broad and evergreen with entire margins and pinnate-camptodromous venation.

In view of the size and evolutionary development of the family, and the habit, habitat, and geographical distribution of its members, it is surprising that the Annonaceae is so prorly represented or recorded in the fossil record. LaMotte (1952) lists fossil representation for only four of the 130 extant genera. It seems likely that much of this lack of fossil representation is due to the similarity between the leaves of other families of the Magnoliales and those of the Annonaceae.

The Lauraceae and Magnoliaceae, for example, have similar appearing leaves and relativelv good fossil records.

Cleared leaves representing 104 genera and over 500 species of the Annonaceae were examined. In nearly all instances, leaves from 10 percent or more of the species of a genus were cleared.

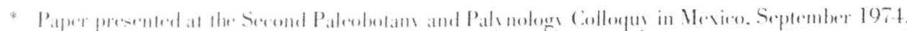

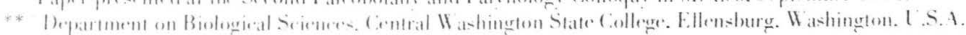

Klucking EP. 1977. Deliberations on leaf venation as supported by the Annonaceae. Boletín de la Sociedad Botánica de México 36: 21-31. 


\section{SELECTED FEATURES OF VENATION}

One of the major problems in characterizing venation patterns is that of determining what features are significant. Constantin von Ettingshausen (1861) described and illustrated over 200 characters of leaf venation using the leaves of various species of dicotyledones to typify the occurrence of diverse combinations of features. However, the paleobotanist is interested in the relationship of fossil leaves to those of extant taxa and the genus is the tason most significant in establishing relationships. Therefore, one of the major purposes for studving the extant leaves of the Annonaceare was to characterize the venation patterns of the genera.

Significant differences between genera appear when one considers the nature and associations of five features. Four of these features deal with the secondary veins and the other with the general orientation and state of development of the venation between the secondary veins. Contrasts in the features of secondary veins become more evident when the secondary veins are divided into three segments. The first of these segments is the departure angle segment (DAS) which extends from the point of departure to a distance around $.5 \mathrm{~cm}$ laterally. In leaves over $10 \mathrm{~cm}$ in length, the DAS mav extend out to $1 \mathrm{~cm}$. Secondary veins in the leaves of the Annonaceae continue laterally beyond the DAS to a point, at varying distances inside the leaf margin, where they branch. One branch generally the weater of the two. is directed basally and the branch is directed apically. The basally directed branch usually joins the apically directed branch from the secondars rein below thus closing off the area between adjacent secondaries. This enclosed area is termed the intercostal areal. That portion of the secondary veins, bevond the DAS, bounding the intercostal area above and below is termed the lateralary while the marginal houndary of the intercostal area is called the marginalary. Segments of the secondary are shown on figure 1 .

The significant features selected for discussion here are the course of the departure angle segment, the course of the lateralary, the angle the lateralary makes with the midrib of the leaf, the spacing of the secondary veins along the midrib, and the nature and degree of development of the intercostal venation. Examples of these features are shown in figureg $2-15$.

Three general courses of the DAS were noted. One type shows the secondary vein arching basally, along another type the secondary veip is straight or curving gently apically, while in the third type the secondary vein curves abruptly apically after tracing a short straight course. These types are referred to here as Recurvate, Rectilineate-Curvate, and Geniculate respectively.

Lateralary courses may be classified into five categories. These are arched or curved basally, sinuated or zig-zag along an approximately straight line, sigmoid or arched basally then rurved distally, bowed or gently curved distally, and straight.

The angle which the lateralary makes with the midrib can be approximated as high whem it is 60" or more, moderate when it is around $45^{\prime \prime}$, and low when it is $30^{\prime \prime}$ or less. 
Spacing of the secondary veins along the midrib, like the lateralary angles, cannot be precisely measured. Leaves may be considered to have broadly spaced secondaries if the secondaries in the middle part of the leaf are noticeably over one-tenth the lenght of the leaf apart, medium spacing if they are around one-tenth the length of the leaf apart, and narrow spacing if they are noticeably less than one-tenth of the lenght of the leaf apart.

Of all of the features selected for description, the nature and development of the intercostal venation is the most difficult to describe. For purposes of discussion, the intercostal area is divided into two sectors, an inner or medial half - the mesiad - and an outer or marginal half - the laterad. Within the intercostal area, the descriptive aspect of venation is imparted by the nature and relationships of the intersecondary veins and the tertiary veins. Intersecondary veins range from subsecondary to subtertiary in strength and arise directly from the primary vein. They extend varying distances laterally, generally tending to parallel the secondary veins. Usually when intersecondary veins are present. more than one occurs within a given intercostal area and the major one occurs midway between the secondaries and expends further laterally than the others in that intercostal area. These features are shown on fig. 14.

Tertiary veins commonly cross the intercostal area transversely from secondary vein to secondary vein or they branch back toward the midrib from the marginalary. This type of ramification is called admedial and is also frequently exhibited by branches from the intersecondary veins.

The pattern of venation within the intercostal area is either oriented predominantly in a lateral direction or transversely. Leaves in which the tendency is laterally usually have intersecondaries extending into the laterad and connecting with the marginalary or branches from the marginalary or branches from the marginalary extend admedially with ramifications to connect with the midrib. This laterally dominant pattern is called medialate in the former example and marginalate in the latter. In many instances, laterally dominant intereostal venation will be a combination of the two types.

The transverse pattern of intercostal venation is one in which there is a tendency for the tertiary weins to be directed in apical and basal directions and thus connect with adjacent secondary veins. The ultimate pattern of this type within the Annonaceae is one in which there are 10.15 transverse tertiary reins and no intersecondaries or if intersecondaries are present they are involved in a "webbing" in the axils of the secondary veins. The tendency tow ard the realization of this pattern is termed costulation. Costulation may progress from the laterad tow ard and into the mesiad or from the mesiad, between the secondary and intersecondary veins toward 1 is - laterad. In many instances it is again a combination of the two. Frequently after wntulation has reached a 3.5 transverse costulate stage, further costulation occurs lhw...h the deselopment of veins arising from the tertiarv veins and paralleling them to connect with the secondary veins. 


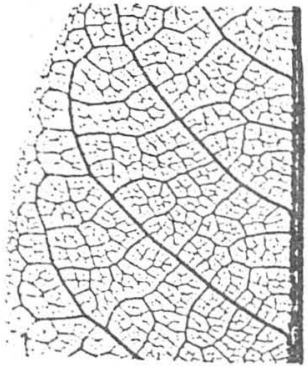

Figure 1

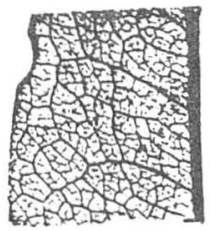

Figure 2

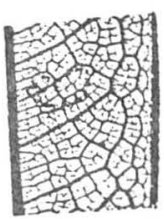

Figure 3

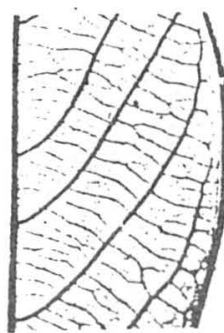

Figure 4

Figure 1. Portion of leaf showing segments of secondary rein

a. Departure Angle Segment (DAS)

b. Lateralary

c. Marginalary

Figure 2. Portion of leaf showing Recurvate DAS

Figure 3: Portion of leaf showing Rectilineate-Curvate DAS

Figure 4. Portion of leaf showing Geniculate DAS

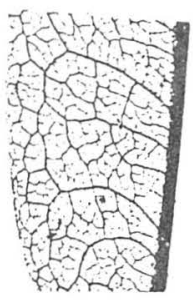

Figure 5

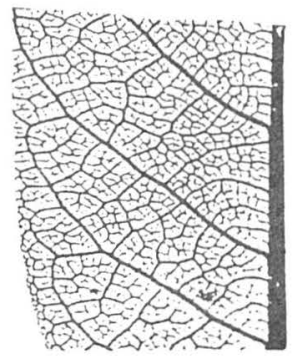

Figure 6

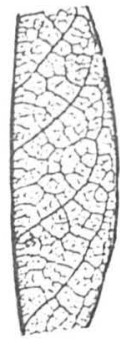

Figure 7

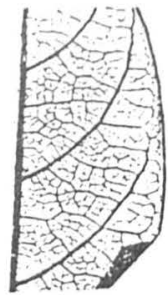

Figure 8

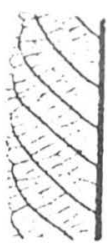

Figure 9

Figure 5. Portion of leaf showing arching lateralaries Figure 6. Portion of leaf showing sinuate lateralaries Figure 7. Portion of leaf showing sigmoid lateralaries Figure 8. Portion of leaf showing bowed lateralaries Figure 9. Portion of leaf showing straight lateralaries 


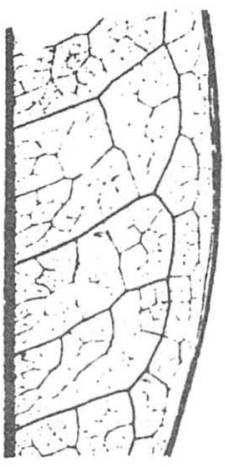

Figure 10

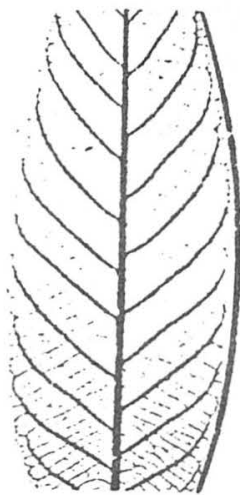

Figure 11

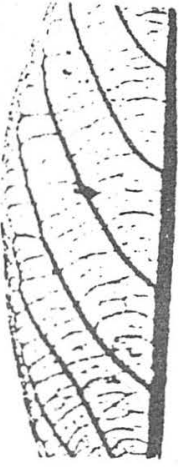

Figure 12

Figure 10. Leaf portion showing broad spacing of secondaries and lateralaries at low angles.

Figure 11. Leaf portion showing medium spaced lateralaries at moderate angles.

Figure 12. Leaf portion showing medium spaced secondaries at low angles.

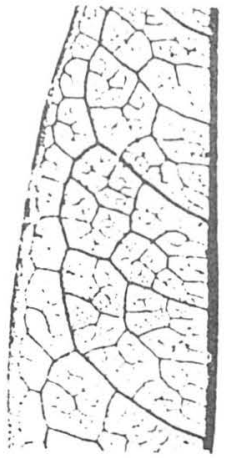

Figure 13

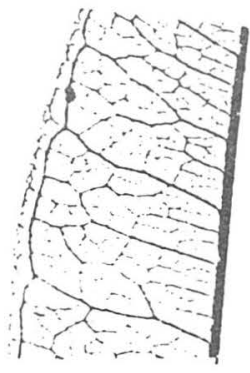

Figure 14

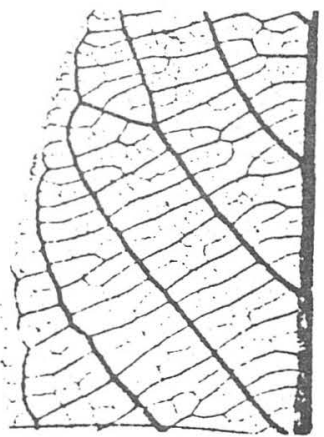

Figure 15

Figure 13. Portion of leaf showing marginalate lateralate intercostal venation.

Figure 14. Portion of leaf showing medialate lateralate intercostal venation.

Figure 15. Portion of leaf showing costulate intercostal venation with "webbing". 


\section{SELECTED PATTERNS OF VENATION}

Another problem in characterizing patterns of venation involves the variation in venation which exists within a single leaf, the several leaves of the various species of a genus.

One a single leaf, the venation in the basal portion of the leaf is distinet from that of the median and apical parts. Within the Annonaceae, the venation in the basal portion of the leaf appears to develop first and is undeveloped while that in the apical part of the leaf develops last and is underdeveloped. The venation in the area of the greatest width of the leaf nearly always shows the maximum development and consistency for any given leaf and so the features in this area are those which are used for description.

Variations in venation within the species is treated much the same as variation iin renation within a single leaf. Among the leaves of a species there are some which have undeveloped renation. some with underdeveloped venation, and many with maximum development. The latter serve as representatives of the species and are determined by subjective examination of numerous leaves rather than by extensive measurements. Amonge the Annonareae the venation pattern of the species is relatively uniform and variations appear to be due largely to modifications in leaf shape.

The differences in the appearance of venation patterns among the leaves of the species of a genus posses a much more difficult problem. One cannot rule out the misidentifications of specimens in the herbaria but misidentifications cannot account for the majority of variation. Clues to an approach to the solution of this problem are suggested by Wernham (cited by Cronquist, 1968). He states that, "the general relation between the significant features of the ancestry and those of the descendants is, that in the former the characters in question are not constant throughout the group, nor may they be completely evolved. In other words, we are dealing with tendencies to characters and not with the critical characters themselves...." Utilizing this idea and assuming that the development of leaf venation proceeds from a condition of irregular or disorganized venation to one of relatively organized or uniform venation, one may proceed to deal with variation in the venation patterns of a genus. Organized venation may be distinguished from disorganized venation by the occurrence of an increased number of similar venation units oriented in the same direction.

On the basis of the general course of the DAS the leaves of the various genera of the Annonaceae have been placed into three large groups. These groups are the Recurvate group, the Rectilineate-Curvate group, and the Geniculate group. In term- if numbers of genera, the Recurvate and Geniculate groups are roughly equal and about one-third larger than the Rectilineate-Curvate group. However, the genera included within the Rectilineate-Curvate group have more than twice as many species as are included in each of the other groups. Characterizations of the venation patterns of a genus from each of these groups will illustrate the manner in which the family has been treated as a whole. 


\section{KLUCKING, LEAF VENATION IN ANNONACEAE}

Xilopia, with 170 species, is the largest genus within the Recurvate group. It is also the only genus within the family to have pantropic distribution. Leaves of 23 of the 170 extant species were cleared and examined. The leaf venation of the genus may be described as follows:

Secondary veins with recurvate to rectilineate-curvate DAS; lateralaries recurved. straight or slightly curved, often dichotomously branching at closure; lateralary angle generally high to moderate; spacing irregularly broad to medium; intersecondaries always present, commonly dichotomously branched; costules transverse with abcostules tending to be dominant and tertiaries from the marginalary generally admedially oriented; intercostal venation lateralatemarginalate or lateralate costulate.

Within the genus there are at least three phases of venational development. Xylopis Inxifolia (figure 16) represents the stage of least development, $X_{y}$ lopis quintasii (figure

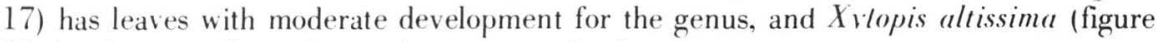
18) has leaves with maximum development for the genus.

The leaves of Xilopia buxifolia have lateralary courses which are arched or sinuated and terminated by dichotomous branching. In most instances, the basally directed segment is much more branched and developed than the apical segment. The secondaries are broal to medium and irregularly spaced at high angles. They show some crude costulation in the laterad but for the most part, the intercostal venation is oriented in a laterad fashion due to admedial ramifications of the costules.

The leaves of Xilopia quintusii show a phase of greater development. While there is little or no difference in the spacing of the lateralaries or the angles they make with the midrib, their courses are sinuated to straight and the marginalary overtops the branch forming closure. In other words, the secondary vein is sympodially branchea in this species rather than dichotomously branched. Dichotomous branching on leaves of Yilopia quinlasii is nicely shown by the intersecondaries and branches from these are directed admedially. The nature of the intercostal venation is similar to that of $X$. luxifolin but more lateralate in aspect and more uniform.

Venation patterns of the leaves of Xylopia altissima are much more developed. The secondaries are svmpodially branched with the lateralaries curved and at moderate angles. The lateralaries are also irregularly broadly to medially spaced. Costulation dominates the laterad of the intercostal areas with intersecondaries somewhat infrequent and restricted to the mesiad. Dichotomous branching is most prevalent among the tertiary veins.

Leaves of the first two species of Xylopia mentioned above are at various phases of development toward the realization of the pattern represented by Xylopia altissima. The apparent tendencies in this sequence are toward the costulation of the intercostal area, a restricting of dichotomous branching to higher order of venation, and a lowering of the lateralary angles. There are several other lines of development noted among the leaves of Xvlopia which will not be discussed here.

The genus Rollinin was selected to represent the Rectilineate-Curvate group. This 


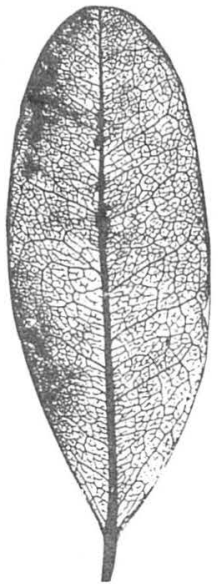

Figure 16

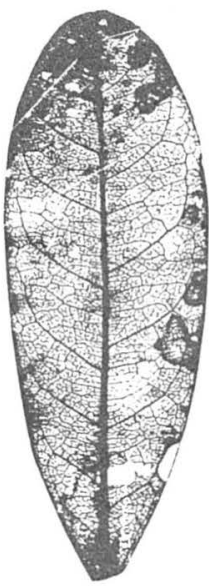

Figure 17

Figure 16. Cleared leaf of $X$ ylopia buxifolia Baill.

Figure 17. Cleared leaf of $X$ ylopia quintasii Pierre ex. Engl \& Diels.

genus is composed of 65 species with distribution in the neotropics. Ten species of the genus were cleared and examined and two of these. Rollinia multiflora (Figure 19) and R. jimenezii (Figure 20) were chosen for discussion. The venation pattern fo the genus may be characterized as follows:

Secondary veins with Rectilineate-Curvate DAS; lateralaries straight to slightly curved, sympodial; lateralary angles generally around $60^{\prime \prime}$ with spacing medium to somewhat narrow; intersecondaries commonly restricted to the mesiad or involved in "webbing"; intercostal venation lateralate in the inner part of the mesiad and the rest of the area is costulate.

The leaves of Rollinia jimenezii have intercostal areas which are costulated to a much greater degree than those of $R$. multiflora and the lateralaries tend to be bowed (monopodial) rather than sinute (sympodial). Within the leaves of $R$. mulliflor there is evidence of dichotomous branching along the marginalary portions of the secondaries and on the intersecondaries while in the leaves of $R$. jimenezii dichotomous branching is mainly restricted to the tertiary veins and those of higher order. In comparison with the leaves of $X \%$ opia, a greater degree of uniformity and regularity in renation is readily apparent. The species of the genus Rollinia, in general, do not have the variability exhibited by the species of Xilopia. 


\section{KLUCKING, LEAF VENATION IN ANNONACEAE}

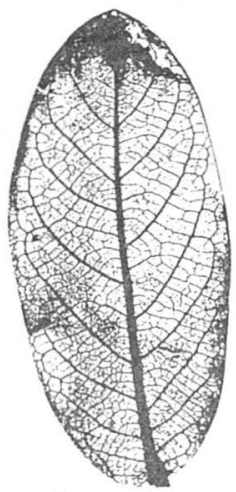

Figure 18

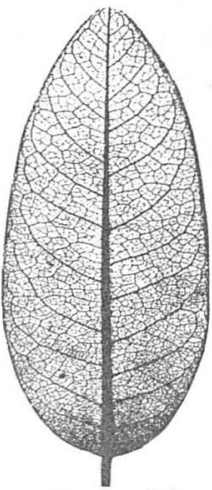

Figure 19

Figure 18. Cleared specimen of Xilopia allissima Boerl.

Figure 19. Cleared specimen of Rollinia multiflora Spligt.

The leaves of Friesodielsin were selected to represent the venational patterns of the geniculate group. Members of this group have the greatest development of venation within the Annonaceale. This genus consists of 55 species with distribution in tropical Asia and Africa. Leaves of 12 species, 4 with African distribution and 8 from the Asiatic tropies were cleared and examined. Most of the leaves of species of this genus were relatively uniform in venational patterns. A specimen of Friesodielsia acuminata (Figure 21) was chosen to show the pattern of development. Characterization of the venation pattern of the genus is as follows:

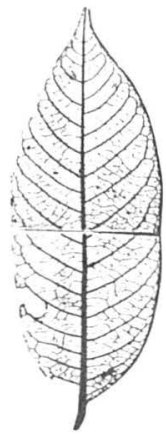

Figure 20

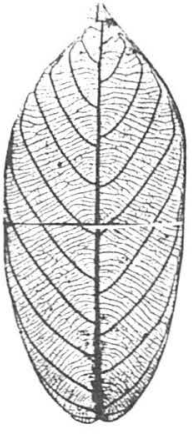

Figure 21

Figure 20. Cleared leaf of Rollinio jimenezii Safford.

Figure 2l. (Cleared leaf of Friesodiclsia acuminata (Merr.) Sincl. 


\section{BOLETIN DE LA SOCIEDAD BOTANICA DE MEXICO No. 36, 1977}

Secondary veins with Geniculate DAS; lateralaries straight though some may be slightly bowed; spacing of secondaries medium, relatively regular, lateralaries at low angles; marginalaries sympodial, forming $60^{\circ}$ or greater angles with the lateralaries in the upper part of the leaf; intercostal venation costulate, consisting of 10 or more transverse costules and "webbing" in the axils of the secondary veins.

The pattern exhibited by $F$. acuminata is quite uniform. Further development of venation within leaves having reached this stage appears to be tending toward the formation of a greater degree of costulation through the formation of new costules between the existing ones.

\section{CONCLUSIONS AND SUMMARY}

Extensive studies of the leaves of the Annonaceae indicates that the genera of this family can be characterized by describing the nature and degree of the development of the venation patterns of the species of the genus. The variation in the venation patterns of the species of a genus represent different phases of development in the lines or tendencies toward the realization of a characteristic pattern of venation. Large genera (usually those with 75 species or more) show greater variation in venation patterns than smaller genera and some reveal more than one line or trend.

Development of venation proceeds from irregular or disorganized patterns to regular or organized patterns. An irregular pattern consists of one in which there are many different kinds of venational units oriented in varying directions, while an organized pattern consists of an increase in the number of similar venational units oriented in the same direction.

Many of the changes towards increased organization are reflected in the courses of the secondary veins. Recurvate departure angle segments of secondary veins are commonly found with broad spacing of the secondaries, high angled lateralaries, numerous intersecondaries, and irregular intercostal venation. These features are considered to be primitive within the family. Advanced features consist of a Geniculate DAS, medium or narrow spacing of the secondaries, low angled lateralaries, and uniform, generally costulate intercostal venation with few or no intersecondaries.

Lateralaries and marginalaries tend to follow the stages of branching outlined in the Telome theory. They progress from dichotomous branching to sympodial to monopodial in different phases of development of venation patterns. Related to this progression is the occurence of dichotomous branching in the intersecondaries when the lateralaries have reached the svmpodial phase or showing a restriction of dichotomous branching to the tertiary and higher order veins when the main intersecondaries are sympodial in nature. 


\section{KLUCKING, LEAF VENATION IN ANNONACEAE}

The appearance and concurrence of these different features suggests that certain prevailing trends occur in the development of venation patterns. Perhaps the most noticeable of these trends are the lowering of the lateralary angle. the narrowing of the spare between secondary veins. and the costulation of the intercostal area. These trends and the various phases of venation development can be readily detected and-described without exhaustive measurements or extensive terminology. It remains to be determined whether or not the number of features utilized here are sufficient to delimit most or all of the genera of woody dicots.

\section{BIBLIOGRAPHY}

CRONQUIST. A., 1968. The Evolution and Classification of Flonering Plants. Houghton Mifflin Co. Boston.

ETTINGSHAUSEN, C. V. 1861. Die Blall-Sterele der Dikothledonen. Druck and Verlag der Kais. Kön. Hof-und Staatsdruckerei, Wien.

LAMOTTE. R. S. 1952. Catalogue of the Cenozoic plants of North America through 1950. Geol. Sor. Amer. Hem. 51 .

WALKER. J. W...1971. Pollen Morphologv, Phytogeography, and Phylogeny of the Annonaceate. Conlr. (iras Herb. 202: 1-130.

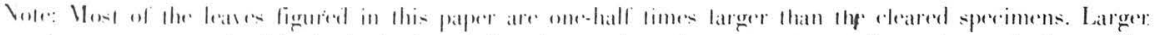

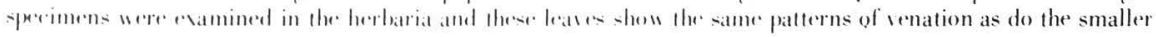
antes. 